\title{
Multifunctional Nanotherapeutics: "The Engines of Healing”
}

\section{Jagat R. Kanwar*}

Laboratory of Immunology and Molecular Biomedical Research, Centre for Biotechnology and Interdisciplinary Biosciences, Institute for Technology and Research Innovation, Deakin University, Geelong, Australia

The occurrence of cancer is well parameterized in a more systemic fashion due to abalative derailing of numerous cell signalling paradigms ultimately triggering explosive chaotization in cellular growth patterns $[1,2]$. The ingenious complexity in a bunch of intricate cancer signalling mechanisms along with prominent tumour survival mechanisms including anti apoptotic proteins (survivin, livin, hypoxia, heat shock proteins etc), oncogenic signalling, tumour suppressor mechanisms have imposed a major cessation for the therapeutic success of current chemo and radiological treatment regimes thereby promoting drug resistant cancers [3-7]. To address these concerns, nanotechnological based strategies had recently conquered therapeutic ground. Nanoscale entities tagged with functional tumour specific molecules such as receptor-mediated tumour ligands, aptamers, locked nucleic acid conjugates (LNA), RNA interference technology, microRNA, siRNA, peptidomemitics, antibodies, combinationatorial therapeutic formulations, drug encapsulation and imaging probes are deemed to be relatively smaller than the cancer cell itself and hence, targeted drug delivery with enhanced selectivity, therapeutic synergism can be achieved [8-10]. The dramatic outreach of multifunctional nanotherapeutic approaches have been exploited in the area of cancer diagnostics by utilisation of economically viable nanomicrofludic lab on chip devices promoting personalised cancer nanomedicine and in the area of therapeutic imaging by utilisation of state of art imaging technologies including fluorescent imaging strategies, magnetic resonance imaging (MRI), positron emission tomograpy (PET), single photon emission computed tomograpy (SPECT), photodynamic laser therapy (PDT) [11]. The major concerns arising due to prominent side effects associated with conventional therapeutics had forced the scientific community to explore natural product derived therapeutics including medicinal herbs, curcumin, lactoferrin, neem etc for the anticancer ability [5]. Surprisingly, these natural therapeutic entities revealed their promising capability in bursting out the nodal cancer signalling mechanisms causing remarkable inhibition in cancer progression [12].

Multifunctional nanodrug delivery systems were fabricated with natural product derived therapeutics. Polymer based formulations with drug encapsulated in alginate coated nanocarriers (ACNC) and alginate gel encapsulated chitosan ceramic nanocore nanocarriers caused enhanced mitochondrial tumour apoptosis leading to survivin down regulation [1]. Another set of completely variable natural product based super paramagnetic iron oxide nanoparticle (SPIONS) nanotherapeutic formulational were devised in a combinatorial strategy coupling with tumour targeting entities including epithelial cell adhesion molecule (EPCAM), aptamer variants in combination with locked nucleic acid conjugates including LNA - nucleolin aptamer, LNA - EPCAM aptamer [10]. These multifunctional nanotherapeutics exhibited predominant anticancer activity both in vitro and in vivo $[8,10]$. Furthermore, advanced near infrared fluorescence in vivo imaging studies were conducted to delineate effective localisation patterns and anti tumour activity of drug coupled SPIONS [1]. Studies to extrapolate the theranostic abilities of nano drug delivery system utilising MRI, SPECT, PDT and SPECT imaging modalities are under progression [1].

In conclusion, multifunctional nanotherapeutic approaches can offer lucerative advantages in the realms of cancer diagnosis, targeted therapy and advanced therapeutic imaging capabilities $[2,13]$. However, there is an inherent necessity to further channelize the developments in the era of nanomedicine along with unison of upcoming technologies including computational biology, systems biological engineering approach in order to reap maximum benefits from so called nanotherapeutics - "the engines of healing" as phrased and speculated by Erix Drexel in his notable work "the engines of creation" $[2,14]$.

\section{References}

1. Kanwar JR, Kamalapuram SK, Kanwar RK (2012) Survivin Signaling in Clinica Oncology: A Multifaceted Dragon. Med Res Rev.

2. Kreeger PK, Lauffenburger DA (2010) Cancer systems biology: a network modeling perspective. Carcinogenesis 31: 2-8.

3. Kanwar RK, Cheung CH, Chang JY, Kanwar JR (2010) Recent advances in anti-survivin treatments for cancer. Curr Med Chem 17: 1509-1515.

4. Kanwar JR, Kamalapuram SK, Kanwar RK (2011) Targeting survivin in cancer: the cell-signalling perspective. Drug Discov Today 16: 485-494.

5. Kanwar JR, Kamalapuram SK, Kanwar RK (2010) Targeting survivin in cancer: patent review. Expert Opin Ther Pat 20: 1723-1737.

6. Kanwar JR, Singh N, Kanwar RK (2011) Role of nanomedicine in reversing drug resistance mediated by ATP binding cassette transporters and P-glycoprotein in melanoma. Nanomedicine (Lond) 6: 701-714.

7. Kanwar JR, Shen WP, Kanwar RK, Berg RW, Krissansen GW (2001) Effects of survivin antagonists on growth of established tumors and B7-1 immunogene therapy. J Natl Cancer Inst 93: 1541-1552.

8. Kanwar JR, Kanwar RK, Mahidhara G, Cheung CH (2012) Cancer Targeted Nanoparticles Specifically Induce Apoptosis in Cancer Cells and Spare Normal Cells. Aust J Chem 65: 5-14

9. Kanwar JR, Mahidhara G, Kanwar RK (2011) Antiangiogenic therapy using nanotechnological-based delivery system. Drug Discov Today 16: 188-202.

${ }^{*}$ Corresponding author: Dr. Jagat R. Kanwar, PhD, Associate Professor of Immunology and Cell Biology, Laboratory of Immunology and Molecular Biomedical Research, Centre for Biotechnology and Interdisciplinary Biosciences, Institute for Technology and Research Innovation, Deakin University, Geelong, Australia, Tel: 00613 52271148; Fax: 00613 52272539; E-mail: Jagat.Kanwar@deakin.edu.au

Received October 04, 2012; Accepted October 10, 2012; Published October 12 2012

Citation: Kanwar JR (2012) Multifunctional Nanotherapeutics: "The Engines of Healing". J Cancer Sci Ther 4: xxiii-xxiv. doi:10.4172/1948-5956.1000e115

Copyright: (c) 2012 Kanwar JR. This is an open-access article distributed unde the terms of the Creative Commons Attribution License, which permits unrestricted use, distribution, and reproduction in any medium, provided the original author and source are credited. 
Citation: Kanwar JR (2012) Multifunctional Nanotherapeutics: “The Engines of Healing”. J Cancer Sci Ther 4: xxiii-xxiv. doi:10.4172/19485956.1000 e115

10. Kanwar JR, Roy K, Kanwar RK (2011) Chimeric aptamers in cancer celltargeted drug delivery. Crit Rev Biochem Mol Biol 46: 459-477.

11. Kievit FM, Zhang M (2011) Cancer nanotheranostics: improving imaging and therapy by targeted delivery across biological barriers. Adv Mater 23: $\mathrm{H} 217-\mathrm{H} 247$.

12. Kanwar JR, Palmano KP, Sun X, Kanwar RK, Gupta R, et al. (2008) 'Iron- saturated' lactoferrin is a potent natural adjuvant for augmenting cancer chemotherapy. Immunol Cell Biol 86: 277-288.

13. O'Shea P (2012) Future medicine shaped by an interdisciplinary new biology. Lancet 379: 1544-1550.

14. Drexler KE (1986) Engins of Creation: The Coming Era of Nanotechnology, (Anchor Books) 